\title{
Nonuniform Image Representation in Area-of-Interest Systems
}

\author{
Natan Peterfreund and Yehoshua Y. Zeevi
}

\begin{abstract}
This paper is concerned with image representation by data distributed nonuniformly and in particular with a representation scheme suitable for "area-of-interest" imaging. The class of signals under consideration, whose information density varies with position, can be represented according to a nonuniform sampling scheme. Position-varying projection operators are presented as simple low-pass filtering operations in a Fourier-like domain. Sequential projections are used for pyramidal representation of nonuniformly sampled images. It is shown that irregular random sampling, prevents, under certain mild restrictions, aliasing effects.
\end{abstract}

\section{INTRODUCTION}

Tras N A VARIETY of applications of image technology such as wide field-of-view sensing, communications, and display systems, which are designed to exploit the advantages inherent in the structural organization and functional characteristics of the human visual system, it is desirable to present an image by a set of nonuniformly distributed samples.

Studies of the structure of the sampling grid of the human eye have revealed inhomogeneities in the distribution of cones over the retina. While the receptors density is uniform over the center of the fovea, it monotonically decreases as a function of eccentricity [6], [9]. This principle of nonuniform allocation of computational resources is further elaborated in later stages of visual processing [8]. The problem of aliasing that may arise due to low-density distribution of cones over the retina is prevented, according to Yellot [15], [16], by the irregularities in the receptors location.

In the biological visual system, the optics of the eye processes the image nonuniformly [9] by projecting it (in both mathematical and physical sense of projection) to prevent aliasing effects that may arise due to nonuniform distribution of cones over the retina. In the present study, we elaborate a projection technique to be used for image conditioning prior to the application of nonuniform sampling. Based on the studies of Clark et al. [3], Porat and Zeevi [12], Zeevi et al. [18], and Zeevi and Shlomot [19], we elaborate a formalism for projection suitable for nonuniform sampling and processing of images. We show that suboptimal sampling treated as

Manuscript received December 16, 1992; revised March 15, 1995. The associate editor coordinating the review of this paper and approving it for publication was Prof. Avideh Zakhor.

N. Peterfreund is with the Armament Development Authority, Haifa, Israel.

Y. Y. Zeevi is with the Department of Electrical Engineering, Technion-Israel Institute of Technology, Haifa, Israel.

IEEE Log Number 9413974. irregularities in the locations of the sampling points prevents, under some mild restrictions, aliasing effects such as jaggedness and/or appearance of phantom images. A Fourier-like transform to be presented simplifies the projection of images under consideration, similarly to the role of the conventional Fourier transform in the case of bandlimited functions. We show that nonlinear projection operations degenerate in this "compatible domain" (in the sense of Zadeh's compatible transform) to simple linear lowpass filtering operations. Finally, we generalize the pyramidal approach [2], [11] and apply it to images sampled nonuniformly both in Cartesian and polar coordinate systems.

\section{PReliminaries}

In this section, we review the essentials of the classical sampling theorem and its extension to nonuniform sampling of class of functions that are not bandlimited. For further detail, see [3], [5], [19].

Given a vector $x \in \mathbb{R}^{n}$ and a matrix $A$, let $x^{t}$ and $|A|$ denote the transpose of $x$ and the determinant of $A$, respectively.

For $\mathbf{x} \triangleq\left[x_{1}, x_{2}\right]^{t}$ and $\omega \triangleq\left[w_{1}, w_{2}\right]^{t}$, let $g(\mathbf{x})$ and $G(\boldsymbol{\omega})$ be a 2-D signal and its Fourier transform. Suppose that $G(\boldsymbol{\omega})=0$ outside a bounded region $\Omega$ and

$$
G(\boldsymbol{\omega})=\sum_{\mathbf{k}} G(\boldsymbol{\omega}-\mathbf{U k}) \quad \forall \boldsymbol{\omega} \in \Omega \quad\left(\mathbf{k} \in \mathbb{Z}^{2}\right)
$$

for some nonsingular matrix $\mathbf{U}$. Under these conditions, $g(\mathbf{x})$ can be reconstructed from its samples on a uniform grid according to the formula [5]

$$
g(\mathbf{x})=|\operatorname{det} \mathbf{V}| \sum_{\mathbf{n}} g\left(\mathbf{x}_{\mathbf{n}}\right) \Phi(\mathbf{x}-\mathbf{V n}) \quad\left(\mathbf{n} \in \mathbb{Z}^{2}\right)
$$

where $\mathbf{V}$, which is the sampling matrix, satisfies $\mathrm{U}^{t} \mathbf{V}=2 \pi I$ and

$$
\Phi(\mathbf{x}) \triangleq \frac{1}{4 \pi^{2}} \iint_{\boldsymbol{\Omega}} \exp \left(j \omega^{t} \mathbf{x}\right) d \boldsymbol{\omega}
$$

A function $g(\mathbf{x})$ whose Fourier transform vanishes outside a finite region $\Omega$ is said to belong to $B^{\Omega}$.

Let $\boldsymbol{\gamma}(\mathbf{x}) \triangleq\left[\gamma_{1}(\mathbf{x}), \gamma_{2}(\mathbf{x})\right]^{t}$ be a continuous function called a distortion function and $\boldsymbol{\gamma}_{\mathbf{x}}(\mathbf{x})$ the corresponding Jacobian matrix. We require that $\gamma(0)=0,\left|\boldsymbol{\gamma}_{\mathbf{x}}(\mathbf{x})\right|>0$ for all $\mathbf{x}$ and $\|\boldsymbol{\gamma}(\mathbf{x})\| \rightarrow \infty$ as $\|\mathbf{x}\| \rightarrow \infty$ so that the inverse function 
$\boldsymbol{\gamma}^{-1}(\mathbf{x})$ exists. The space $B_{\boldsymbol{\gamma}(\mathbf{x})}^{\Omega}$, which is called the space of locally bandlimited functions, is defined as follows [19]:

$$
f(\mathbf{x})=g[\boldsymbol{\gamma}(\mathbf{x})] \text { where } g(\mathbf{x}) \in B^{\Omega} \Leftrightarrow f(\mathbf{x}) \in B_{\boldsymbol{\gamma}(\mathbf{x})}^{\boldsymbol{\Omega}} \text {. }
$$

It can be readily verified that $g(\mathbf{x}) \in L^{2}\left(\mathbb{R}^{2}\right)$ iff for $f(\mathbf{x})=$ $g[\boldsymbol{\gamma}(\mathbf{x})]$

$$
\iint f^{2}(\mathbf{x}) d \boldsymbol{\gamma}(\mathbf{x})<\infty
$$

The space of functions that satisfy (4) will be denoted by $L^{2} \boldsymbol{\gamma}(\mathrm{x})\left(\mathbb{R}^{2}\right)$. According to (1), a function $f(\mathbf{x}) \in B_{\boldsymbol{\gamma}(\mathbf{x})}^{\boldsymbol{\Omega}}$ can be reconstructed from its samples on a nonuniform grid $\mathbf{x}_{\mathbf{n}}=\boldsymbol{\gamma}^{-1}(\mathbf{V n})$ according to [19]:

$$
f(\mathbf{x})=|\operatorname{det} \mathbf{V}| \sum_{\mathbf{n}} f\left(\mathbf{x}_{\mathbf{n}}\right) \Phi(\boldsymbol{\gamma}(\mathbf{x})-\mathbf{V n}) .
$$

The operator that projects a given signal $w(\mathbf{x})$ into the space of locally bandimited functions $B_{\boldsymbol{\gamma}(\mathbf{x})}^{\boldsymbol{\Omega}}$ is [19]

$$
f(\mathbf{x})=\iint_{-\infty}^{\infty} w(\boldsymbol{\xi}) \Phi(\boldsymbol{\gamma}(\mathbf{x})-\boldsymbol{\gamma}(\boldsymbol{\xi})) d \boldsymbol{\gamma}(\boldsymbol{\xi})
$$

where $\Phi(\cdot)$ is given by (2). This operation, which is used for signal conditioning prior to the application of nonuniform sampling $\mathbf{x}_{\mathbf{n}}=\boldsymbol{\gamma}^{-1}(\mathbf{V} \mathbf{n})$, is an extension of the conventional lowpass filtering and is used as a first step in applying a nonuniform sampling scheme.

\section{PROJECTION INTO THE SPACE OF LOCALLY BANDLIMITED FUNCTIONS}

\section{A. A Fourier-Like Characterization of Signals and Systems}

Similar to Fourier analysis and synthesis of bandlimited functions, it is of interest to find a compatible domain in which signals from $B_{\boldsymbol{\gamma}(\mathbf{x})}^{\boldsymbol{\Omega}}$ can be analyzed and synthesized in a natural manner.

Consider a 2-D position-varying system characterized by a superposition integral [17]:

$$
y(\mathbf{x})=\iint f(\boldsymbol{\xi}) h(\mathbf{x}, \boldsymbol{\xi}) d \boldsymbol{\xi}
$$

where $f(\mathbf{x})$ and $y(\mathbf{x})$ are the system's input and output, respectively, and $h(\mathbf{x}, \boldsymbol{\xi})$ is a position-varying impulse response. Define

$$
F_{\boldsymbol{\gamma}}(\boldsymbol{\omega}) \triangleq \iint f(\mathbf{x}) e^{i \boldsymbol{\omega}^{t} \boldsymbol{\gamma}(\mathbf{x})}\left|\boldsymbol{\gamma}_{\mathbf{x}}(\mathbf{x})\right| d \mathbf{x}
$$

and

$$
f(\mathbf{x}) \triangleq \frac{1}{4 \pi^{2}} \iint F_{\boldsymbol{\gamma}}(\omega) e^{-i \boldsymbol{\omega}^{t} \boldsymbol{\gamma}(\mathbf{x})} d \boldsymbol{\omega}
$$

as the Fourier-like transform of $f(\mathbf{x})$ and the corresponding inverse transformation. Define

$$
H_{\boldsymbol{\gamma}}(\boldsymbol{\omega}, \mathbf{x}) \triangleq \iint h(\mathbf{x}, \boldsymbol{\xi}) e^{i \boldsymbol{\omega}^{t}[\boldsymbol{\gamma}(\mathbf{x})-\boldsymbol{\gamma}(\boldsymbol{\xi})]} d \boldsymbol{\xi}
$$

as the system's position-varying transfer function. Substituting (8)-(10) into (7), we obtain the superposition integral as a filtering operation in the Fourier-like domain:

$$
y(\mathbf{x})=\frac{1}{4 \pi^{2}} \iint_{D} F_{\boldsymbol{\gamma}}(\boldsymbol{\omega}) H_{\boldsymbol{\gamma}}(\boldsymbol{\omega}, \mathbf{x}) e^{-i \boldsymbol{\omega}^{i} \boldsymbol{\gamma}(\mathbf{x})} d \boldsymbol{\omega}
$$

where $D$ is the appropriate support in the Fourier-like domain. An important case of the position-varying transfer function $H_{\boldsymbol{\gamma}}(\boldsymbol{\omega}, \mathbf{x})$ is independent of $\mathbf{x}$. Given the system (7), a transformation (10) that satisfies this condition is said to be a compatible transform with respect to the particular system (7) [4].

Lemma 3.1: The transformation given by (8)-(11) is compatible with respect to system (7) iff

$$
h(\mathbf{x}, \boldsymbol{\xi})=h_{o}(\boldsymbol{\gamma}(\mathbf{x})-\boldsymbol{\gamma}(\boldsymbol{\xi}))\left|\boldsymbol{\gamma}_{\mathbf{x}}(\boldsymbol{\xi})\right|
$$

for some $h_{o}(\cdot) \in L^{2}\left(\mathbb{R}^{2}\right)$.

Proof: Substituting (12) into (10), we have

$$
H_{\boldsymbol{\gamma}}(\boldsymbol{\omega}, \mathbf{x})=\iint h_{0}(\boldsymbol{\gamma}(\mathbf{x})-\boldsymbol{\gamma}(\boldsymbol{\xi})) e^{i \boldsymbol{\omega}^{t}[\boldsymbol{\gamma}(\mathbf{x})-\boldsymbol{\gamma}(\boldsymbol{\xi})]} d \boldsymbol{\gamma}(\boldsymbol{\xi})
$$

where $d \boldsymbol{\gamma}(\boldsymbol{\xi})=d \boldsymbol{\xi}\left|\boldsymbol{\gamma}_{\mathbf{x}}(\boldsymbol{\xi})\right|$. Let $\mathbf{z} \triangleq \boldsymbol{\gamma}(\mathbf{x})-\boldsymbol{\gamma}(\boldsymbol{\xi})$, and then

$$
H_{\boldsymbol{\gamma}}(\boldsymbol{\omega}, \mathbf{x})=\iint h_{o}(\mathbf{z}) e^{i \boldsymbol{\omega}^{i} \mathbf{z}} d \mathbf{z}=H_{o}(\boldsymbol{\omega})
$$

where $H_{o}(\boldsymbol{\omega})$ is the Fourier transform of $h_{o}(\cdot)$. Since

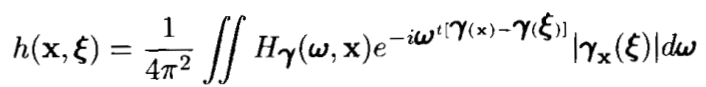

the converse statement can be readily proven.

Theorem 3.1: For any signal $f(\mathbf{x}) \in L_{\boldsymbol{\gamma}(\mathbf{x})}^{2}\left(\mathbb{R}^{2}\right)$ and a transfer function $H_{o}(\boldsymbol{\omega})$, the Fourier transform of $h_{o}(\cdot)$, which is bandlimited in a finite region $\Omega^{\prime} \subseteq \Omega$, the solution of (11) ((7)) belongs to $B_{\boldsymbol{\gamma}(\mathbf{x})}^{\boldsymbol{\Omega}}$. If $H_{\mathrm{o}}(\boldsymbol{\omega})=1$ in $\Omega$ and zero elsewhere, then (11) defines the projection operator into $B_{\boldsymbol{\gamma}(\mathbf{x})}^{\boldsymbol{\Omega}}$.

Proof: Let $G(\boldsymbol{\omega})=F_{\boldsymbol{\gamma}}(\boldsymbol{\omega}) H_{o}(\boldsymbol{\omega})$, where $F_{\boldsymbol{\gamma}}(\boldsymbol{\omega})$ is a Fourier-like transform of the input signal $f(\mathbf{x})$. Since $G(\boldsymbol{\omega})$ is bandlimited in $\Omega$, by (11), we have $y(\mathbf{x})=g[\boldsymbol{\gamma}(\mathbf{x})] \in B_{\boldsymbol{\gamma}(\mathbf{x})}^{\Omega}$, where $g(\cdot)$ is the inverse Fourier transform of $G(\boldsymbol{\omega})$. For $f(\mathbf{x}) \in B_{\boldsymbol{\gamma}(\mathbf{x})}^{\boldsymbol{\Omega}}$, however, $F_{\boldsymbol{\gamma}}(\boldsymbol{\omega})$ is bandlimited in $\Omega$. It follows that if $H_{o}(\boldsymbol{\omega})=1$ in $\Omega$ and zero elsewhere, then

$$
F_{\boldsymbol{\gamma}}(\boldsymbol{\omega}) H_{o}(\boldsymbol{\omega})=F_{\boldsymbol{\gamma}}(\boldsymbol{\omega})
$$

and $y(\mathbf{x})=f(\mathbf{x})$ in (11) as asserted.

The latter result generalizes the results of [19] concerning the projection into $B_{\boldsymbol{\gamma}(\mathbf{x})}^{\boldsymbol{\Omega}}$. In fact, for the special case where $H_{o}(\boldsymbol{\omega})=1$ on $\Omega$ and zero elsewhere, the superposition integral (7) degenerates to the projection operator (6) given by Zeevi and Shlomot [19].

A special case of interest is the projection into "subband"like regions. This projection, as will be shown, serves as a first step in a sampling and decimation procedure applied to functions that belong to $B_{\gamma(x)}^{\Omega}$ space. In the following, we shall assume that $\Omega$ is a bounded region that contains the origin. The projected functions may then be viewed as lowpass versions of the original signals with a Fourier-like support 
$\boldsymbol{\Omega}^{\prime} \subset \boldsymbol{\Omega}$. In the sequel, we show the correspondence between the bandwidth of $g(\mathbf{x}) \in B^{\Omega}$ and the distortion function $\gamma(\mathbf{x})$ in the $B_{\boldsymbol{\gamma}(\mathbf{x})}^{\boldsymbol{\Omega}}$ space.

Lemma 3.2: Consider the bounded region $\Omega \subset \mathbb{R}^{2}$ that contains the origin. For $m, n \geq 1$

$$
\boldsymbol{\gamma}_{m n}(\mathbf{x}) \triangleq\left[\gamma_{1}(\mathbf{x}) / m, \quad \gamma_{2}(\mathbf{x}) / n\right]^{t}
$$

and

$$
\Omega_{m n} \triangleq\left\{\begin{array}{lll}
1 & \text { if } & {\left[m \omega_{1}, n \omega_{2}\right]^{t} \in \Omega} \\
0 & \text { otherwise }
\end{array}\right.
$$

we have

$$
B_{\boldsymbol{\gamma}(\mathbf{x})}^{\boldsymbol{\Omega}_{m n}}=B_{\boldsymbol{\gamma}_{m n}(\mathbf{x})}^{\boldsymbol{\Omega}} .
$$

Proof: Suppose that $f(\mathbf{x})=g\left[\boldsymbol{\gamma}_{m n}(\mathbf{x})\right]$, where $g(\mathbf{x}) \in$ $B^{\Omega}$. Let $w\left(x_{1}, x_{2}\right)=g\left(\frac{x_{1}}{m}, \frac{x_{2}}{n}\right)$. Since $w\left(x_{1}, x_{2}\right) \in B^{\boldsymbol{\Omega}_{m n}}$ and $f(\mathbf{x})=w[\boldsymbol{\gamma}(\mathbf{x})]$, it follows that $f(\mathbf{x}) \in B_{\boldsymbol{\gamma}(\mathbf{x})}^{\boldsymbol{\Omega}_{m n}}$, and hence, $B_{\boldsymbol{\gamma}_{m n}(\mathbf{x})}^{\boldsymbol{\Omega}} \subset B_{\boldsymbol{\gamma}(\mathbf{x})}^{\boldsymbol{\Omega}_{m n}}$. The converse statement can be readily proved.

According to the above result, the projection operator into $B_{\boldsymbol{\gamma}_{m n}(\mathbf{x})}^{\boldsymbol{\Omega}}$ is given by the simple lowpass filter

$$
H_{\boldsymbol{\gamma}}(\boldsymbol{\omega}, \mathbf{x})=H_{m n}(\boldsymbol{\omega}) \triangleq\left\{\begin{array}{ccc}
1 & \text { if }\left[m \omega_{1}, n \omega_{2}\right]^{t} \in \boldsymbol{\Omega} \\
0 & \text { otherwise. }
\end{array}\right.
$$

Hence, signals from $B_{\boldsymbol{\gamma}_{m n}(\mathbf{x})}^{\boldsymbol{\Omega}}$ for $m, n>1$ may be treated in the $B_{\boldsymbol{\gamma}(\mathbf{x})}^{\boldsymbol{\Omega}}$ space as signals that are bandlimited in $\boldsymbol{\Omega}_{m n} \subset \boldsymbol{\Omega}$

In certain representations [3], [7] via simple variable changes, signals in the $B_{\boldsymbol{\gamma}(\boldsymbol{x})}^{\Omega}$ space are characterized as having position-varying bandwidth. In this representation, the projection operator can be viewed as a lowpass filtering operation to a position-dependent band-area $\Omega(x)$. This area is a position-dependent scaled version of $\boldsymbol{\Omega}$.

Let $\boldsymbol{\gamma}(\mathbf{x})=\left[\mathbf{w}_{1}(\mathbf{x}) x_{1}, \mathbf{w}_{2}(\mathbf{x}) x_{2}\right]^{t}$ and suppose that $F_{\boldsymbol{\gamma}}(\boldsymbol{\omega})$ is bandlimited to $\Omega$. Substituting $\gamma(\mathbf{x})$ into (9), we have

$$
f(\mathbf{x})=\frac{1}{4 \pi^{2}} \iint_{\boldsymbol{\Omega}} F_{\boldsymbol{\gamma}}(\boldsymbol{\omega}) e^{-i \boldsymbol{\omega}^{t}\left[\mathbf{W}_{1}(\mathbf{x}) \boldsymbol{x}_{1}, \mathbf{W}_{2}(\mathbf{x}) x_{2}\right]^{t}} d \boldsymbol{\omega} .
$$

For $\bar{\omega} \triangleq\left[\overline{\omega_{1}}, \overline{\omega_{2}}\right]^{t} \triangleq\left[\mathrm{w}_{1}(\mathbf{x}) \omega_{1}, \mathbf{w}_{2}(\mathbf{x}) \omega_{2}\right]^{t}, f(\mathbf{x})$ takes the form

$$
f(\mathbf{x})=\iint_{\boldsymbol{\Omega}(\mathbf{x})} F_{\boldsymbol{\gamma}}\left(\frac{\overline{\omega_{1}}}{\mathrm{w}_{1}(\mathbf{x})}, \frac{\overline{\omega_{2}}}{\mathrm{w}_{2}(\mathbf{x})}\right) e^{-i \overline{\boldsymbol{\omega}}^{t} \mathbf{x}} \frac{d \overline{\boldsymbol{\omega}}}{\mathrm{w}_{1}(\mathbf{x}) \mathrm{w}_{2}(\mathbf{x})}
$$

where

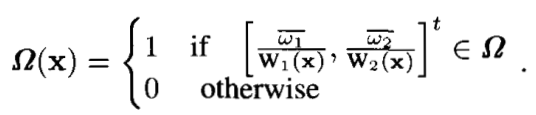

This region, which is denoted as the focal band-area of $f(\mathbf{x})$, is given by scaling $\Omega$ along the frequency axes by $\mathrm{w}_{1}(\mathbf{x})$ and $\mathrm{w}_{2}(\mathbf{x})$, respectively. Thus, locally, a function $f(x) \in B_{\boldsymbol{\gamma}(\mathbf{x})}^{\boldsymbol{\Omega}}$ can be interpreted as having a local band-area $\Omega(x)$. Using this representation, the projection from $B_{\boldsymbol{\gamma}(\mathbf{x})}^{\Omega}$ into $B_{\boldsymbol{\gamma}}^{\Omega}(\mathbf{x})$ can be viewed, according to Lemma 3.2, as lowpass filtering a signal with local band-area $\Omega(\mathbf{x})$ to a local band-area
$\Omega_{m n}(\mathbf{x}) \subset \Omega(x)$, which is given by scaling $\mathrm{w}_{1}(\mathbf{x})$ and $\mathrm{w}_{2}(\mathbf{x})$ in (15) to become $\frac{W_{1}(x)}{m}$ and $\frac{W_{2}(x)}{n}$, respectively. For the special case where

$$
\boldsymbol{\gamma}(\mathbf{x})=\left[\mathbf{w}(\|\mathbf{x}\|) x_{1}, \mathbf{w}(\|\mathbf{x}\|) x_{2}\right]^{t}
$$

we have the local band area $\Omega(\mathbf{x})=\mathrm{w}(\|\mathrm{x}\|) \Omega$, which is a function of the radial distance from the origin. Obviously, in this case $\|\boldsymbol{\gamma}(\mathbf{x})\|=\sqrt{\gamma_{1}^{2}(\mathbf{x})+\gamma_{2}^{2}(\mathbf{x})}=\gamma(r)$, where $r=\|\mathbf{x}\|$.

\section{B. Discrete Implementation}

Consider the projection from $B_{\boldsymbol{\gamma}^{\prime}(\mathbf{x})}^{\Omega}$ space into $B_{\boldsymbol{\gamma}(\mathbf{x})}^{\Omega}$ for some distortion functions $\boldsymbol{\gamma}^{\prime}(\mathbf{x})$ and $\boldsymbol{\gamma}(\mathbf{x})$. Let $f(\mathbf{x}) \in B_{\boldsymbol{\gamma}^{\prime}(\mathbf{x})}^{\boldsymbol{\Omega}}$ be given by its values on the sampling grid $\mathbf{x}_{\mathbf{k}}=\gamma^{\prime-1}(\mathbf{V k})$, where, by (1) and (5), $V$ is the sampling matrix of the unwarped grid that prevents aliasing. Substituting (5) with $\boldsymbol{\gamma}(\mathbf{x})=\boldsymbol{\gamma}^{\prime}(\mathbf{x})$ into (6), the discrete projection operator of $f(\mathbf{x}) \in B_{\boldsymbol{\gamma}^{\prime}(\mathbf{x})}^{\boldsymbol{\Omega}}$ into $B_{\boldsymbol{\gamma}(\mathbf{x})}^{\boldsymbol{\Omega}}$ is given by [18]

$$
y(\mathbf{x})=\sum_{\mathbf{k}} f\left(\mathbf{x}_{\mathbf{k}}\right) C_{\mathbf{k}}(\mathbf{x}) \quad \mathbf{k} \in \mathbb{Z}^{2}
$$

where

$$
C_{\mathbf{k}}(\mathbf{x}) \triangleq \int_{-\infty}^{\infty} \Phi\left(\boldsymbol{\gamma}^{\prime}(\boldsymbol{\xi})-\mathbf{V k}\right) \Phi(\boldsymbol{\gamma}(\mathbf{x})-\boldsymbol{\gamma}(\boldsymbol{\xi})) d \boldsymbol{\gamma}(\boldsymbol{\xi}) .
$$

The projected function $y(\mathbf{x})$ can be reconstructed from its samples on the grid $\mathbf{x}_{\mathbf{n}}=\boldsymbol{\gamma}^{-1}(\mathbf{V n})$ according to (5). Although the above equation considers the projection between two distinct function spaces, a special case of interest is the projection into a "subband"-like region, i.e., the projection from $B_{\boldsymbol{\gamma}(\mathbf{x})}^{\boldsymbol{\Omega}}$ into $B_{\boldsymbol{\gamma}_{m \eta}(\mathbf{x})}^{\boldsymbol{\Omega}}$, where $\boldsymbol{\gamma}_{m n} \triangleq\left[\gamma_{1}(\mathbf{x}) / m, \gamma_{2}(\mathbf{x}) / n\right]^{t}$ and $\boldsymbol{\Omega}$ contains the origin. This projection, as noted, serves as a first step in a decimation procedure. According to Lemma 3.2 , the corresponding continuous projection operator is given by (11) with the filter (14). Substituting (5) into (8), we have

$$
\begin{aligned}
F_{\boldsymbol{\gamma}}(\boldsymbol{\omega})= & |\operatorname{det} \mathbf{V}| \sum_{\mathbf{k}} f\left(\mathbf{x}_{\mathbf{k}}\right) \\
& \cdot \iint \Phi(\boldsymbol{\gamma}(\mathbf{x})-\mathbf{V k}) e^{i \boldsymbol{\omega}^{t} \boldsymbol{\gamma}(\mathbf{x})}\left|\boldsymbol{\gamma}_{\mathbf{x}}(\mathbf{x})\right| d \mathbf{x} \\
= & |\operatorname{det} \mathbf{V}| \sum_{\mathbf{k}} f\left(\mathbf{x}_{\mathbf{k}}\right) e^{i \boldsymbol{\omega}^{t} \mathbf{V k}} H_{o}(\boldsymbol{\omega})
\end{aligned}
$$

where $H_{o}(\boldsymbol{\omega})=1$ in $\Omega$ and zero elsewhere. Substituting $F_{\boldsymbol{\gamma}}(\boldsymbol{\omega})$ into (11), with $H_{\boldsymbol{\gamma}}(\boldsymbol{\omega}, \mathbf{x})=H_{m n}(\boldsymbol{\omega})$ given by (14), the projection operator into $B_{\boldsymbol{\gamma}_{m n}(\mathbf{x})}^{\boldsymbol{\Omega}}$ is

$$
y(\mathbf{x})=|\operatorname{det} \mathbf{V}| \sum_{\mathbf{k}} f\left(\mathbf{x}_{\mathbf{k}}\right) \Phi_{m n}(\boldsymbol{\gamma}(\mathbf{x})-\mathbf{V k})
$$

where $\Phi_{m n}(\cdot)$ is the inverse Fourier transform of $H_{m n}(\boldsymbol{\omega})$. Since $y(\mathbf{x}) \in B_{\boldsymbol{\gamma}_{m \eta}(\mathbf{x})}^{\boldsymbol{\Omega}}$, by (5), it can be reconstructed from its samples on a grid that satisfies the condition

$$
\left[\frac{\gamma_{1}\left(\mathbf{x}_{\mathbf{k}}\right)}{m}, \frac{\gamma_{2}\left(\mathbf{x}_{\mathbf{k}}\right)}{n}\right]^{t}=V \mathbf{k} \quad\left(\mathbf{k} \in \mathbb{Z}^{2}\right)
$$




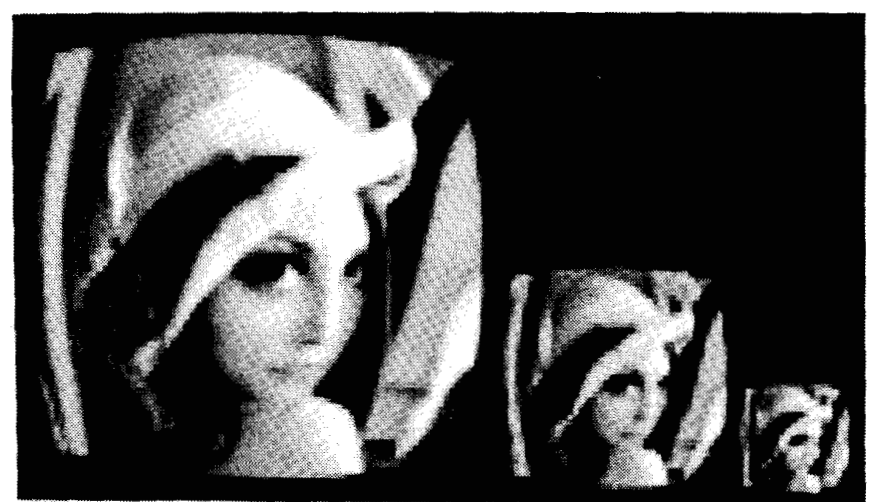

Fig. 1. First three levels of a pyramid of images sampled nonuniformly along Cartesian coordinates. The images are shown distorted into uniform grids. The reference image (level 1 ) is of $80 \times 80$ pixels, and each higher level is a decimated version of the previous one.

or, equivalently

$$
\left[\gamma_{1}\left(\mathbf{x}_{\mathbf{k}}\right), \gamma_{2}\left(\mathbf{x}_{\mathbf{k}}\right)\right]^{t}=\left[\begin{array}{cc}
m & 0 \\
0 & n
\end{array}\right] \mathbf{V k} .
$$

Hence, the projection operator permits decimation of the sequence along the sampling grid by $1: m$ and $1: n$ along the $x_{1}$ and $x_{2}$ axes, respectively. Note that in the special case where $\gamma_{1}(\mathbf{x})$ and $\gamma_{2}(\mathbf{x})$ are linear functions of $x_{1}$ and $x_{2}$, respectively, this procedure degenerates to the conventional decimation process of bandlimited functions.

Example 3.1-Sampling in Cartesian Coordinates: Let $B^{\circ}$ denote the space of all functions that are bandlimited to the radius $\pi$. A function $f(\mathbf{x})$ will be said to belong to $B_{\gamma(\mathbf{x})}^{o}$ iff there exists $g(\mathbf{x}) \in B^{o}$ such that $f(\mathbf{x})=g[\gamma(\mathbf{x})]$. By (1), the appropriate sampling matrix that would prevent aliasing will be the identity matrix. Alternatively, one can use a hexagonal sampling matrix

$$
\mathbf{V}=\left[\begin{array}{cc}
1 / \sqrt{3} & 1 / \sqrt{3} \\
1 & -1
\end{array}\right]
$$

Such a sampling scheme is more efficient and requires $13.4 \%$ fewer samples than the rectangular [5]. Using (2), we have

$$
\Phi(\mathbf{x})=\frac{\pi}{2} \operatorname{Jic}(\pi\|\mathbf{x}\|)
$$

where $\|\mathbf{x}\|=\sqrt{x_{1}^{2}+x_{2}^{2}}$ and $\operatorname{Jic}(a) \triangleq \frac{J_{1}(a)}{a}\left(J_{1}(a)\right.$ is the first-order Bessel function of the first kind). According to (5), the interpolation formula of $f(\mathbf{x}) \in B_{\gamma(\mathbf{x})}^{o}$ is then given by

$$
f(\mathbf{x})=|\operatorname{det} \mathbf{V}| \frac{\pi}{2} \sum_{\mathbf{n}} f\left(\mathbf{x}_{\mathbf{n}}\right) J i c(\pi\|\boldsymbol{\gamma}(\mathbf{x})-\mathbf{V n}\|)
$$

where $\mathbf{x}_{\mathbf{n}}=\boldsymbol{\gamma}^{-1}(\mathbf{V n})$. The projection of $f(\mathbf{x}) \in B_{\boldsymbol{\gamma}(\mathbf{x})}^{o}$ into $B_{\boldsymbol{\gamma}(\mathbf{x}) / m}^{o}$, by (18), is

$$
y(\mathbf{x})=|\operatorname{det} \mathbf{V}| \frac{\pi}{2 m^{2}} \sum_{\mathbf{n}} f\left(\mathbf{x}_{\mathbf{n}}\right) \operatorname{Jic}\left(\frac{\pi}{m}(\|\boldsymbol{\gamma}(\mathbf{x})-\mathbf{V n}\|)\right) .
$$

Compared with the sampling grid of $f(\mathbf{x}) \in B_{\boldsymbol{\gamma}(\mathbf{x})}^{\boldsymbol{\Omega}}$ given by $\mathbf{x}_{\mathbf{n}}=\boldsymbol{\gamma}^{-1}(\mathbf{V n})$, the set of samples that represents the projected signal is given by $\mathbf{x}_{\mathbf{n}}=\boldsymbol{\gamma}^{-1}(m \mathbf{V n})$. Hence, the projection operator permits decimation of the sequence along both axes of the sampling grid by $1: m$.
Example 3.2-Sampling in Polar Coordinates: Since polar coordinates are defined only on the right half plane, we define an extended polar system over the entire $(r, \theta)$ plane. According to this extension, the values of $f(r, \theta)$ for the left half plane are obtained by $f(r, \theta)=f(-r, \theta+\pi)$. This extension preserves the smoothness property of the original image at the origin. The Fourier transform of this function will be denoted by $F(\rho, \phi)$.

Let $R_{\pi, K}$ denote the class of all functions $f(r, \theta)$, defined on the extended polar system, with a finite Fourier series expansion in $\theta, \sum_{n=-K}^{K} c_{n}(r) e^{-i n \theta}$, and with $F(\rho, \phi)$ that vanishes for $\|\rho\|>\pi$, where $c_{n}(r)$ are the appropriate coefficients, and $K$ is some positive integer. According to this definition, it can be readily shown that the corresponding Fourier transform $F(\rho, \phi)$ vanishes outside the rectangular region $\Omega=[-\pi, \pi] \times[-K, K]$. By (1), a function $f(r, \theta) \in$ $R_{\pi, K}$ can be reconstructed from its samples on the uniform grid with the sampling matrix (see (A1) in [13])

$$
\mathbf{V}=\left[\begin{array}{cc}
1 & 0 \\
0 & \underline{1}
\end{array}\right]
$$

where $W=\frac{2 K+1}{2 \pi}$. Using (2), we have

$$
\Phi(r, \theta)=W \operatorname{sinc}(r) \operatorname{sinc}(W \theta)
$$

where $\operatorname{sinc}(a)=\frac{\sin (\pi a)}{\pi a}$.

A function $\alpha(r, \theta)$ is said to be in $R_{\gamma(r), K}$ iff there exists a function $\psi(r, \theta) \in R_{\pi, K}$ such that $\alpha(r, \theta)=\psi[\gamma(r), \theta]$. We present sampling schemes and projection operations related to functions in the $R_{\gamma(r), K}$ space. The proofs are given in the Appendix.

A function $\alpha(r, \theta) \in R_{\gamma(r), K}$ can be reconstructed from its samples on the sampling grid $\left[r_{n 1}, \theta_{n 2}\right] \triangleq\left[\gamma^{-1}\left(n_{1}\right), n_{2} / W\right]$ according to the formula (see the Appendix)

$$
\begin{gathered}
\alpha(r, \theta)=\sum_{n_{1}=-\infty}^{\infty} \sum_{n_{2}=0}^{2 K} \alpha\left(r_{n 1}, \theta_{n 2}\right) \operatorname{sinc}\left[\gamma(r)-n_{1}\right] . \\
\frac{\sin \left[\pi W\left(\theta-\theta_{n 2}\right)\right]}{(2 K+1) \sin \left[\left(\theta-\theta_{n 2}\right) / 2\right]}
\end{gathered}
$$



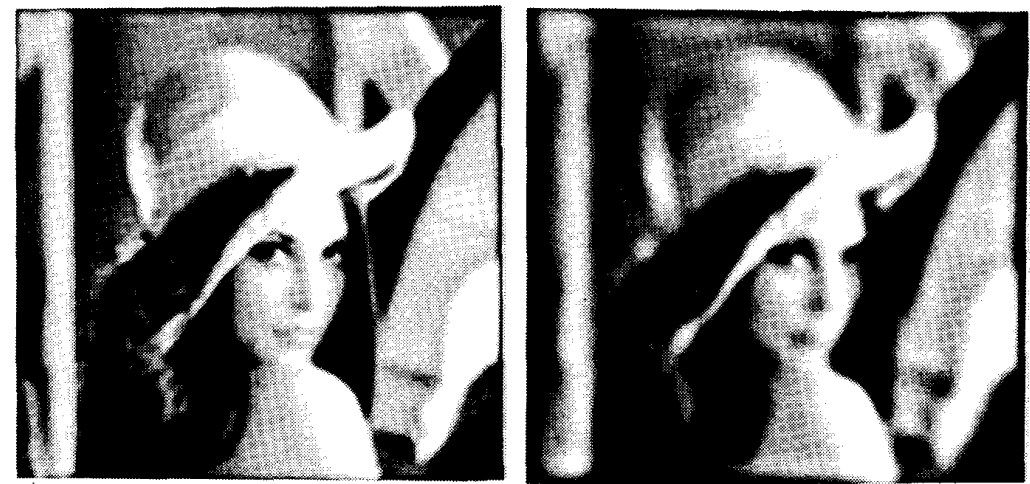

(a)
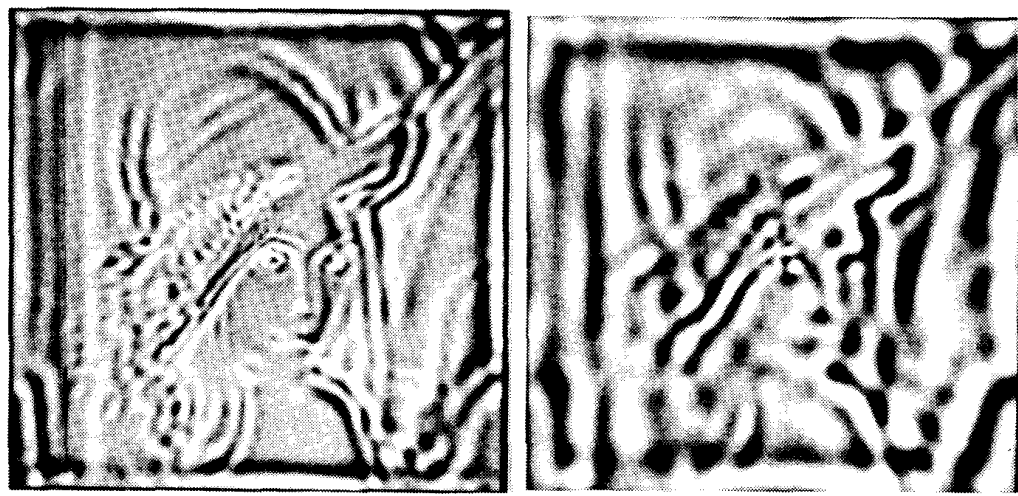

(b)
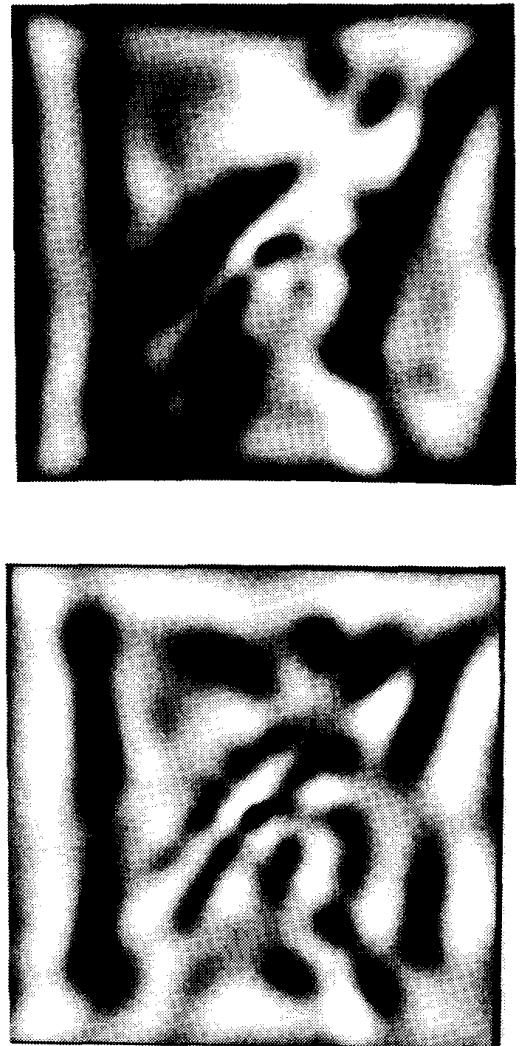

Fig. 2. (a) First three levels of the pyramid shown in Fig. 1 interpolated into $255 \times 255$ pixels; (b) First three levels of "Laplacian-like" pyramid. Each level is the difference between the corresponding level and the higher level of the pyramid shown in (a).

The projection operator of $\eta(r, \theta)$ into $R_{\gamma(r), K}$ is (see the Appendix)

$$
\begin{gathered}
\alpha(r, \theta)=W \int_{-\infty}^{\infty} d \gamma(\rho) \int_{o}^{2 \pi} d \beta\{\eta(\rho, \beta) \operatorname{sinc}[\gamma(r)-\gamma(\rho)] \\
\left.\frac{\sin [\pi W(\theta-\beta)]}{(2 K+1) \sin [(\theta-\beta) / 2]}\right\} .
\end{gathered}
$$

As was noted, the projection (24) may be used as a first step in a decimation procedure. Projection of the image $\alpha(r, \theta) \in$ $R_{\gamma(r), K}$ into $R_{\gamma(r) / n,\lfloor K / m\rfloor}$ (where $\lfloor a\rfloor$ is the integer value of a) can be obtained by substituting (23) into (24). The function $\beta(r, \theta) \in R_{\gamma(r) / n,\lfloor K / m\rfloor}$ is then given by

$$
\begin{gathered}
\beta(r, \theta)=\frac{W_{m}}{n W} \sum_{k=-\infty}^{\infty} \sum_{e=0}^{2 K} \alpha\left(r_{k}, \theta_{e}\right) \operatorname{sinc}[(\gamma(r)-k) / n] . \\
\frac{\sin \left[\pi W_{m}\left(\theta-\theta_{e}\right)\right]}{\left(2 K_{m}+1\right) \sin \left[\left(\theta-\theta_{e}\right) / 2\right]}
\end{gathered}
$$

where $r_{k}$ and $\theta_{e}$ are defined as in (23), $K_{m} \triangleq\lfloor K / m\rfloor$ and $W_{m} \triangleq \frac{2 K_{m}+1}{2 \pi}$. Since $\beta(r, \theta)$ is bandlimited along the $\theta$ variable to the region $[\lfloor-K / m\rfloor,\lfloor K / m\rfloor]$, and since the new distortion function satisfies $\gamma\left(r_{k}\right) / n=k$, the sampling grid of the projected image can be decimated along the $r$ and $\theta$ variables by $1: n$ and $1: m$ (for $K \gg m$ ), respectively.

\section{SyNTHESIS OF IMAGE-PYRAMID GENERATING KERNELS}

Burt and Adelson [2] had constructed an image pyramid by a 2-D decimation processes only in the case of uniform sampling over a Cartesian grid. In the present study, we elaborate on a pyramidal scheme for a variable-resolution image, sampled over either a Cartesian or polar system of coordinates.

\section{A. A Pyramid Scheme in Cartesian Coordinates}

We assume that our 2-D image $f_{1}(\mathbf{x})$ belongs to $B_{\boldsymbol{\gamma}(\mathbf{x})}^{\boldsymbol{\Omega}}$ and is represented as such by a set of samples $\mathbf{x}_{\mathbf{k}}=\boldsymbol{\gamma}^{-1}(\mathbf{V k})$. By projecting it into "half bands," i.e., $B_{\boldsymbol{\gamma}(\mathbf{x}) / m}^{\boldsymbol{\Omega}} m=2,4,8, \cdots$, we get a set of blurred images with variable resolution that can be decimated. Projection into $B_{\boldsymbol{\gamma}(\mathbf{x}) / m}^{\Omega}$ can be accomplished by using (21)

$$
f_{q}\left(\tilde{\mathbf{x}}_{\mathbf{n}}\right)=|\operatorname{det} \mathbf{V}| \frac{\pi}{2 m^{2}} \sum_{\mathbf{k}} f_{1}\left(\mathbf{x}_{\mathbf{k}}\right) \operatorname{Jic}\left(\frac{\pi}{m}\|\mathbf{V}(m \cdot \mathbf{n}-\mathbf{k})\|\right)
$$

where $\tilde{\mathbf{x}}_{\mathbf{n}} \triangleq \boldsymbol{\gamma}^{-1}(m \mathbf{V n})$ is a decimated sampling sequence that represents $f_{q}(\mathbf{x})$ and $q \triangleq 1+\log _{2} m$. The set of projected 
images $f_{1}, f_{2}, \cdots f_{n}$ forms a pyramidal representation of an image in the $B_{\boldsymbol{\gamma}(\mathbf{x})}^{\Omega}$ space. Note that the sampling sequence that represents each $f_{i}(\mathbf{x})$ is given by decimating the one of $f_{i-1}(\mathbf{x})$ by $1: 2$ along both axes. The interpolation formula for each level of the pyramid, according to (20), is

$$
f_{q}(\mathbf{x})=|\operatorname{det} \mathbf{V}| \sum_{\mathbf{k}} f_{q}\left(\tilde{\mathbf{x}}_{\mathbf{k}}\right) \Phi_{\mathbf{k}}^{q}(\mathbf{x})
$$

where

$$
\Phi_{\mathbf{k}}^{q}(\mathbf{x}) \triangleq \frac{\pi}{2} J i c\left(\pi\left\|\frac{\boldsymbol{\gamma}(\mathbf{x})}{m}-\mathbf{V k}\right\|\right)
$$

are the basis functions of the $q$ th level. These functions, which vary with position (see, e.g., Fig. 3), appear in different scales, depending on the pyramid level (see, e.g., Fig. 4).

Motivated by the properties of the visual system and by the need for display systems that are matched to our visual requirements, we next elaborate on an image whose resolution is monotonically decreasing as a function of the radial distance from the center of the visual field. For the purpose of illustration, we arbitrarily select the following distortion function:

$$
\boldsymbol{\gamma}(\mathbf{x})=\left[\frac{5 x_{1}}{\|\mathbf{x}\|^{1 / 2}+5}, \frac{5 x_{2}}{\|\mathbf{x}\|^{1 / 2}+5}\right] .
$$

This function is characterized according to (15) and (16) by a local band area proportional to $\mathrm{w}(\|\mathbf{x}\|)=\frac{5}{\|\mathbf{x}\|^{1 / 2}+5}$, which is directly related to the decrease in the image resolution as a function of the radial distance.

The first three levels of a pyramidal representation of an image in $B_{\boldsymbol{\gamma}(\mathbf{x})}^{o}$ are shown in Fig. 1. The first level was obtained by the discrete projection (17) of an image in $B^{o}$. In Fig. 2(a), we present the interpolated version of the first three levels of the pyramid. The position-varying property of the basis function $\Phi_{\mathbf{k}}^{1}(\mathbf{x})$ is illustrated in Fig. 3. It shows, as expected, that the increase in the spread of these functions with the radial distance is inversely proportional to the resultant resolution of the corresponding image. The scale relation between the basis functions in the first three levels of the pyramid is illustrated in Fig. 4.

In order to compress an image of variable resolution, one can use the "Laplacian pyramid" technique [2]. The first three levels of the nonuniform "Laplacian-like" pyramid are shown in Fig. 2(b).

\section{B. A Pyramid Scheme in Polar Coordinates}

The procedure of decimation can also be applied in polar coordinates. Let the image $\beta_{1}(r, \theta) \in R_{\gamma(r), K}$ be represented by a set of samples $\left(r_{n 1}, \theta_{n 2}\right) \triangleq\left(\gamma^{-1}\left(n_{1}\right), \frac{n_{2}}{W}\right)$, where $W=\frac{2 K+1}{2 \pi}$. Using (25), we obtain the formula for projection into $R_{\gamma(r) / m,\lfloor K / m\rfloor}$ (for $K \gg m$ ):

$$
\begin{gathered}
\beta_{q}\left(\tilde{r}_{k}, \tilde{\theta}_{e}\right)=\frac{1}{m^{2}} \sum_{n_{1}=-\infty}^{\infty} \sum_{n_{2}=0}^{2 K} \beta_{1}\left(r_{n 1}, \theta_{n 2}\right) \operatorname{sinc}\left[\left(m k-n_{1}\right) / m\right] \\
\frac{\sin \left[\frac{\pi}{m}\left(m e-n_{2}\right)\right]}{\left(2 K_{m}+1\right) \sin \left[\left(m e-n_{2}\right) / 2 W\right]}
\end{gathered}
$$

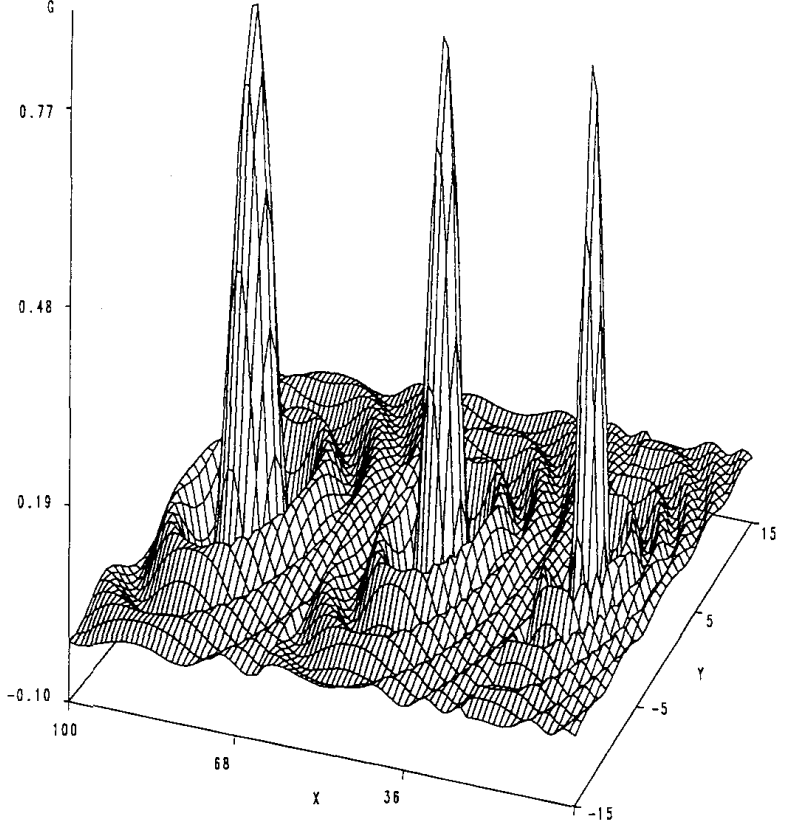

Fig. 3. Increase in speed of the basis function $\Phi_{k}^{1}(x)$ as a function of the distance from the origin for $k=[10,0]^{t},[20,0]^{t}$, and $[30,0]^{t}$.

where the sampling set $\left\{\tilde{r}_{k}, \tilde{\theta}_{e}\right\} \triangleq\left\{\gamma^{-1}(m k), e / W_{m}\right\}$ decimates the original sequence by $m: 1$ along both axes. The variable $q$ specifies the level of the pyramid. According to (23), the interpolation formula for each stage is

$$
\beta_{q}(r, \theta)=\sum_{n_{1}=-\infty}^{\infty} \sum_{n_{2}=0}^{2 K_{m}} \beta\left(\tilde{r}_{k}, \tilde{\theta}_{n 2}\right) \Phi_{n 1, n 2}^{q}(r, \theta)
$$

where

$$
\Phi_{n 1, n 2}^{q}(r, \theta)=\operatorname{sinc}\left[\frac{\gamma(r)}{m}-n_{1}\right] \frac{\sin \left[\pi W_{m}\left(\theta-\tilde{\theta}_{n 2}\right)\right]}{\left(2 K_{m}+1\right) \sin \left[\left(\theta-\tilde{\theta}_{n 2}\right) / 2\right]}
$$

are the basis functions of the $q$ th level. Unlike the basis functions presented in Cartesian coordinates, these functions are position varying only in the $r$ variable.

To illustrate this scheme, we elaborate on an example where the resolution decreases monotonically along $r$ according to the distortion function

$$
\gamma(r)=\frac{5 r}{r^{1 / 2}+5}
$$

The first three levels of the pyramid are shown in Fig. 5. The first level was obtained by a discrete projection of an image in $R_{\pi, K}$. The interpolated images of the first three levels are shown in Fig. 6(a), and the corresponding images of the nonuniform "Laplacian-like" pyramid are shown in Fig. 6(b).

\section{ON IRREGULAR SAMPLING OF LOCALLY BANDLIMITED FUNCTIONS}

Consider an irregular set of sampling points $\left\{\mathbf{x}_{\mathbf{k}}\right\}$ for which the "recovery condition" $\mathbf{x}_{\mathbf{k}}=\boldsymbol{\gamma}^{-1}(\mathbf{V k})$ is not satisfied. Such 


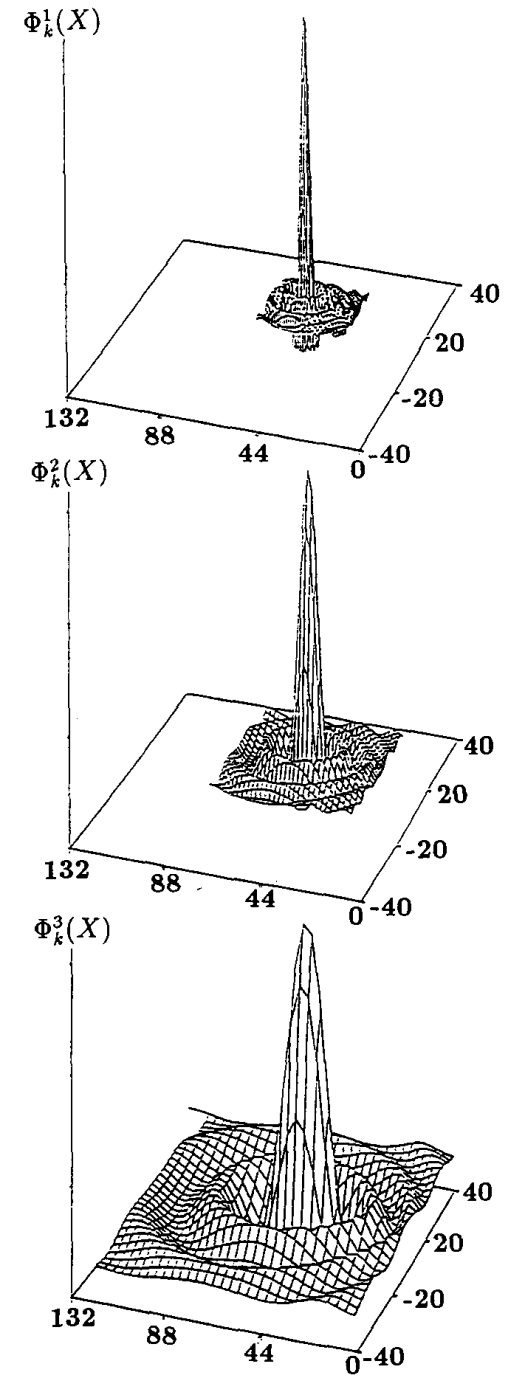

Fig. 4. Scale relation between the basis functions of the first three levels of the pyramid. The upper one corresponds to the first level and the lower one to the third.

a set of sampling points may result from misevaluation of the distortion function $\gamma(\cdot)$ and/or quantization noise.

When a random process $\psi(t)$ with a spectral density $\Psi(\omega)$ bandlimited to $[-\pi, \pi]$ is sampled at unit intervals, the spectral density $\psi(\omega)$ of the sequence $\psi(n)$ satisfies (see, e.g., [1])

$$
\psi(\omega)=\sum_{m} \Psi(\omega-2 \pi n) \quad-\pi \leq \omega \leq \pi .
$$

In such a case, where the spectral density of $\psi(t)$ is directly determined from that of $\{\psi(n)\}$, sampling is said to be aliasfree. Such a condition also holds for deterministic signals with a Fourier transform bandlimited to $[-\pi, \pi]$.

In generalizing the concept of alias-free sampling [10], [1], we next consider the properties of irregular and random sampling in the space of locally bandlimited functions.

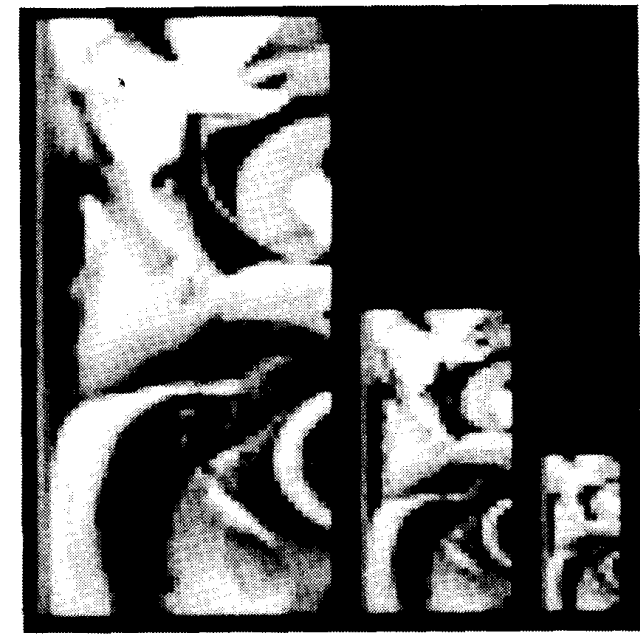

Fig. 5. First three levels of a pyramid of images sampled nonuniformly along polar coordinates. The images are shown distorted into uniform grids. The reference image (level 1 ) is of $80 \times 80$ pixels, and each higher level is a decimated version of the previous one.

\section{A. One-Dimensional Irregular Sampling}

Let $B$ denote the class of functions in $L^{2}(\mathbb{R})$ that are bandlimited to $(-\pi, \pi)$. In analogy to (3), a function $f(t)$ is said to be in $B_{\gamma(t)}$ iff there exists a function $\psi(t) \in B$ such that $f(t)=\psi[\gamma(t)]$.

Let $\hat{F}_{\gamma}$ denote the class of Fourier-like transforms of signals in the $B_{\gamma(t)}$ space. These signals, by (8), are bandlimited to $(-\pi, \pi)$. We next adopt the following definition of alias-free sampling [1], [10].

Definition 5.1: A sampling sequence $\left\{t_{n}\right\}$ is alias-free relative to $\hat{F}_{\gamma}$ if no two functions in $B_{\gamma(t)}$ with different Fourier-like transforms yield the same sampling sequence $\left\{f\left(t_{n}\right)\right\}$.

As a direct consequence of the above definition and the inverse transformation (9), we have the following result.

Lemma 5.1: The sampling sequence $\left\{t_{n}\right\}$ is alias-free relative to $\hat{F}_{\gamma}$ iff for $F_{\gamma}(\omega) \in \hat{F}_{\gamma}$

$\int_{-\pi}^{\pi} F_{\gamma}(\omega) e^{i \omega \gamma\left(t_{n}\right)} d \omega=0$ for all $n$ implies that $F_{\gamma}(\omega) \equiv 0$.

Under the conditions of Lemma 5.1, due to Levinson [10], given some $\epsilon>0$, it is possible to find a polynomial in $\left\{e^{i \omega \gamma\left(t_{n}\right)}\right\}, P_{\epsilon}(\omega)$, such that

$$
\int_{-\pi}^{\pi}\left|F_{\gamma}(\omega)-P_{\epsilon}(\omega)\right|^{2} d \omega<\epsilon .
$$

For the special case where $\gamma\left(t_{n}\right)=n$, we have the Fourier series

$$
\sum_{n=-\infty}^{\infty} a_{n} e^{i n \omega}, \text { where } a_{n}=\frac{1}{2 \pi} \int_{-\pi}^{\pi} F_{\gamma}(\omega) e^{-i n \omega} d \omega
$$

which converges uniformly to $F_{\gamma}(\omega)$. In general, however, this result does not imply that $F_{\gamma}(\omega)$ can be represented by a Fourier-like series of the form $\sum_{n} a_{n} e^{i \omega \gamma\left(t_{n}\right)}$. 

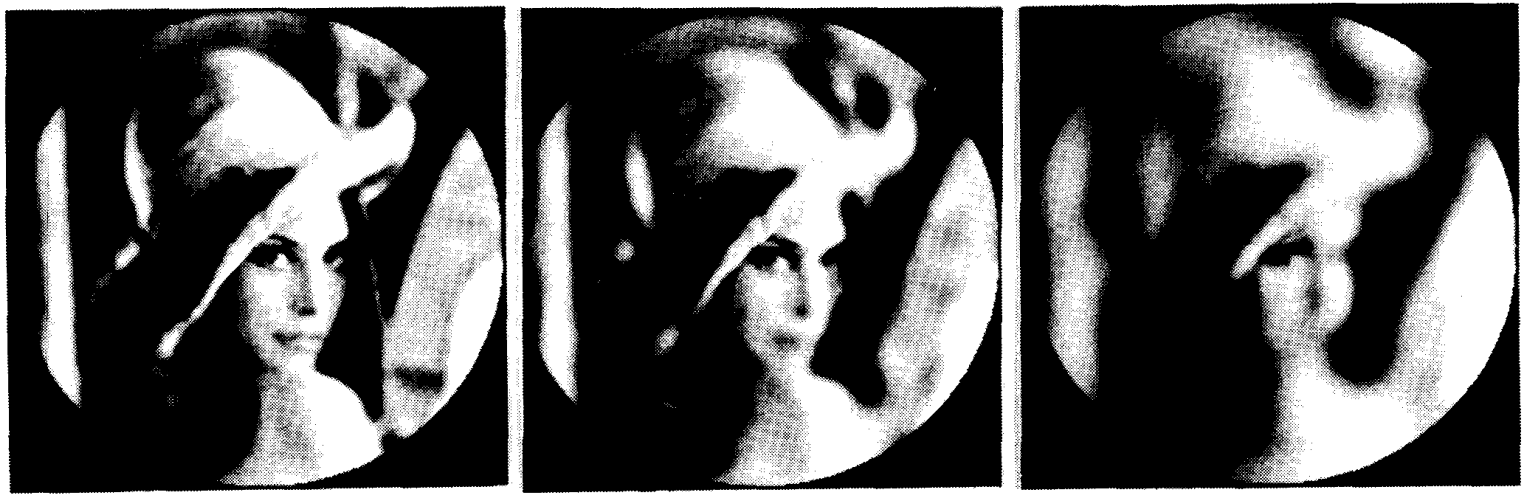

(a)
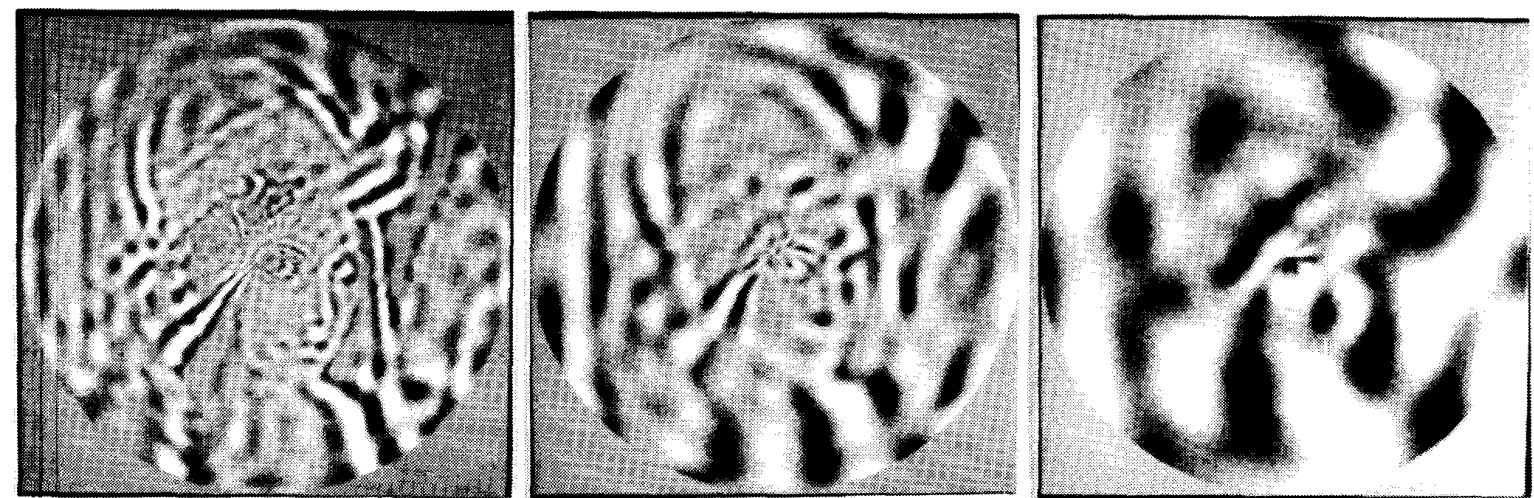

(b)

Fig. 6. (a) First three levels of the pyramid shown in Fig. 5 interpolated into $255 \times 255$ pixels; (b) first three levels of "Laplacian-like" pyramid. Each level is the difference between the corresponding level and the higher level of the pyramid generated over a polar coordinate system.

Lemma 5.2 [10]: If $\left\{t_{n}\right\}$ is a sequence such that

$$
\left|\gamma\left(t_{n}\right)-n\right|<\frac{1}{4} \quad-\infty<n<\infty
$$

the conditions of Lemma 5.1 are satisfied. Furthermore, $\left\{e^{i \omega \gamma\left(t_{n}\right)}\right\}$ incorporates a unique biorthogonal set $\left\{H_{n}(\omega)\right\}$ such that for all $F_{\gamma}(\omega) \in L^{2}(-\pi, \pi)$

$$
\sum_{n=-\infty}^{\infty} a_{n} e^{-i \omega \gamma\left(t_{n}\right),} \text { where } a_{n}=\int_{-\pi}^{\pi} F_{\gamma}(\omega) H_{n}(\omega) d \omega
$$

converges uniformly to $F_{\boldsymbol{\gamma}}(\omega)$ over any interval $(-\pi+\delta \leq$ $\omega \leq \pi-\delta$ ) for any $\delta>0$.

Using the results of Levinson [10], [14], it follows that $\psi(t) \in B$, sampled along the sequence $\left\{\tilde{t}_{n}=\gamma\left(t_{n}\right)\right\}$ that satisfies (26), can be reconstructed from its sampled values according to

$$
\psi(t)=\sum_{n} \psi\left(\gamma\left(t_{n}\right)\right) h_{n}(t)
$$

where $h_{n}(t)$, which is the inverse Fourier transform of $H_{n}(\omega)$, satisfies

$$
h_{n}(t)=\frac{G(t)}{\left[t-\gamma\left(t_{n}\right)\right] G^{\prime}\left(\gamma\left(t_{n}\right)\right)}
$$

and

$$
G(t)=\left[t-\gamma\left(t_{o}\right)\right] \prod_{n=1}^{\infty}\left[1-\frac{t}{\gamma\left(t_{n}\right)}\right]\left[1-\frac{t}{\gamma\left(t_{-n}\right)}\right] .
$$

Note that in the case where $\gamma\left(t_{n}\right)=n$, the interpolating function $h_{n}(t)$ degenerates to the known $\operatorname{sinc}(t-n)$ [10]. According to (27), for $f(t) \in B_{\gamma(t)}$, we finally have the reconstruction formula:

$$
f(t)=\sum_{n} f\left(t_{n}\right) h_{n}(\gamma(t))
$$

where the set of sampling points $\left\{t_{n}\right\}$ satisfies condition (26), and $h_{n}(t)$ is given by (28).

Next, we consider the problem of matching an optimal sampling scheme to some function $f(t) \in B_{\gamma(t)}$ with an unknown distortion function. Let $\hat{\gamma}(t)$ be the estimated $\gamma(t)$ [3], [19] and $\left\{\hat{t}_{n}=\hat{\gamma}^{-1}(n)\right\}$ the corresponding sampling sequence. Using the latter results, it follows that if

$$
\left|\gamma\left(\hat{t}_{n}\right)-n\right|<\frac{1}{4}
$$

the sampling sequence $\left\{\hat{t}_{n}\right\}$ is alias-free with respect to signals in the $B_{\gamma(t)}$ space. Let

$$
\hat{f}(t)=\sum_{n} f\left(\hat{t}_{n}\right) \operatorname{sinc}[\hat{\gamma}(t)-n]
$$


be the reconstruction formula with the estimated distortion function $\hat{\gamma}(t)$. Obviously, we have $\hat{f}\left(\hat{t}_{n}\right)=f\left(\hat{t}_{n}\right)$ and $\hat{f}(t) \rightarrow$ $f(t)$ as $\hat{\gamma}(t) \rightarrow \gamma(t)$.

\section{B. Stochastic Approach}

We denote the class of real stationary random processes with spectral densities bandlimited to $[-\pi, \pi]$ by $\hat{B}$. A real stochastic process is said to be in $\hat{B}_{\gamma(t)}$ iff there exists $\psi(t) \in \hat{B}$ such that $f(t)=\psi[\gamma(t)]$.

Evidently, $f(t) \in \hat{B}_{\gamma(t)}$ is a nonstationary random process whose autocorrelation function $R_{f}\left(t_{1}, t_{2}\right)$ relates to the one of the original processes $R_{\psi}(\cdot)$ by [19]:

$$
\begin{gathered}
R_{f}\left(t_{1}, t_{2}\right) \triangleq E_{f}\left[f\left(t_{1}\right) f\left(t_{2}\right)\right]=E_{\psi}\left[\psi\left(\gamma\left(t_{1}\right)\right) \psi\left(\gamma\left(t_{2}\right)\right)\right]= \\
R_{\psi}\left(\gamma\left(t_{1}\right)-\gamma\left(t_{2}\right)\right) .
\end{gathered}
$$

Consider $R_{f}^{t_{2}}\left(t_{1}\right) \triangleq R_{f}\left(t_{1}, t_{2}\right)$ as a 1-D parametric function. Using the Fourier-like approach, the spectral-like density of $f(t) \in \hat{B}_{\gamma(t)}$ is then defined as

$$
S_{\gamma}(\omega)=\int_{-\infty}^{\infty} R_{f}^{t_{2}}\left(t_{1}\right) e^{i \omega\left[\gamma\left(t_{1}\right)-\gamma\left(t_{2}\right)\right]} \gamma^{\prime}\left(t_{1}\right) d t_{1}
$$

where $\gamma^{\prime}(\cdot)$ is the derivative of $\gamma(\cdot)$. The inverse transformation is given by

$$
R_{f}^{t_{2}}\left(t_{1}\right)=\frac{1}{2 \pi} \int_{-\infty}^{\infty} S_{\gamma}(\omega) e^{-i \omega\left[\gamma\left(t_{1}\right)-\gamma\left(t_{2}\right)\right]} d \omega .
$$

It can be readily verified that for a real stochastic process $f(t) \in \hat{B}_{\gamma(t)}$, the spectral-like density is bandlimited to $[-\pi, \pi]$. In the sequel, we denote the class of spectral-like densities bandlimited to $[-\pi, \pi]$ by $\hat{S}_{\gamma}$.

Next, we extend the concept of alias-free sampling to stochastic processes that belong to the $\hat{B}_{\gamma(t)}$ space. As was noted, a deterministic regular sampling $\left\{t_{n}=n\right\}$ is aliasfree with respect to stochastic processes with spectral densities bandlimited to $[-\pi, \pi]$. Using the spectral-like approach, it can be readily verified that for a distorted sampling sequence $\left\{t_{n}=\gamma^{-1}(n)\right\}$, the spectral-like density of $f(t)$ is directly determined from that of $f\left(t_{n}\right)$. Hence, the latter sampling is alias-free with respect to signals in the $\hat{B}_{\gamma(t)}$ space.

In generalizing the concept of alias-free sampling, we next consider the properties of random sampling sequences. Suppose that $\left\{t_{n}\right\}$ constitutes a point process independent of $f(t)$. The correlation sequence of the discrete parameter process $f\left(t_{n}\right)$ will be denoted by [1]

$$
R\left(t_{m+n}, t_{m}\right)=E\left[f\left(t_{m+n}\right) f\left(t_{m}\right)\right]
$$

in which the expectation is taken over both $f(t)$ and $\left\{t_{n}\right\}$. It is assumed that $\left\{\tau_{n}=\gamma\left(t_{n}\right)\right\}$ constitutes a stationary-point process and that the probability distribution of $\gamma\left(t_{n+m}\right)-$ $\gamma\left(t_{m}\right)$ is independent of $m$. Under these conditions $\left\{f\left(t_{n}\right)=\right.$ $\left.\psi\left[\gamma\left(t_{n}\right)\right]\right\}$ is stationary, so that $R\left(t_{n+m}, t_{m}\right)$ in (31) depends only on $n$ [1].

As a direct extension of the alias-free sampling proposed by Beutler [1], we have the following definition.
Definition 5.2: A sampling sequence $\left\{t_{n}\right\}$ is alias-free relative to $\hat{S}_{\gamma}$ if there do not exist two random processes in $\hat{B}_{\gamma(t)}$ with different spectral-like densities that yield the same correlation sequence $r(n) \triangleq R\left(t_{n+m}, t_{m}\right)$.

In order to have the relation between $r(n)$ and the spectrallike density $S_{\gamma}$ of $f(t)$, we first calculate the expectation (31) over $f$ :

$E_{f}\left[f\left(t_{m+n}\right) f\left(t_{m}\right)\right]=\frac{1}{2 \pi} \int_{-\infty}^{\infty} e^{-i \omega\left[\gamma\left(t_{m+n}\right)-\gamma\left(t_{m}\right)\right]} S_{\gamma}(\omega) d \omega$.

Using the assumptions on $\left\{t_{n}\right\}$, the expected value of $e^{i \omega\left[\gamma\left(t_{m+n}\right)-\gamma\left(t_{m}\right)\right]}$ over $\left\{t_{n}\right\}$ satisfies

$$
\begin{aligned}
E_{t_{n}}\left\{e^{i \omega\left[\gamma\left(t_{n+m}\right)-\gamma\left(t_{m}\right)\right]}\right\} & =E_{\tau_{n}}\left\{e^{i \omega\left(\tau_{n+m}-\tau_{m}\right)}\right\} \\
& =\frac{1}{2 \pi} \int_{-\infty}^{\infty} f_{n}(\tau) e^{i \omega \tau} d \tau=\Phi_{n}(i \omega)
\end{aligned}
$$

in which $f_{n}(\tau)$ is the probability density function for $n$ successive sampling intervals. Substituting (33) and (32) into (31), we have the linear transformation

$$
r(n)=\frac{1}{2 \pi} \int_{-\infty}^{\infty} \Phi_{n}(i \omega) S_{\gamma}(\omega) d \omega .
$$

The alias-free property, by Definition 5.2, suggests that no two different spectral-like densities yield the same correlation sequence $r(n)$. This property is satisfied iff the above transformation is one to one.

Lemma 5.3: The sampling sequence $\left\{t_{n}\right\}$ is alias-free with respect to $\hat{S}_{\gamma}$ iff with $S_{\gamma} \in \hat{S}_{\gamma}$

$$
\int_{\infty}^{\infty} \Phi_{n}(i \omega) S_{\gamma}(\omega) d \omega=0 \text { for all } n \text { implies } S_{\gamma}(\omega) \equiv 0 \text {. }
$$

An example of a sampling sequence that satisfies the conditions of Lemma 5.3 is [1]

$$
\gamma\left(t_{n}\right)=n+u_{n}
$$

where $u_{n}$ are pairwise independent and identically distributed over $\left[-\frac{1}{2}, \frac{1}{2}\right]$. Such a jitter may result from an error in the computed (estimated) distortion function of some distorted-axis stochastic process. If, for example, $\hat{\gamma}(t)$ is the approximated version of $\gamma(t)$, and $t_{n}=\hat{\gamma}^{-1}(n)$ is the corresponding sampling sequence, then under (34), $\left\{t_{n}\right\}$ is alias-free with respect to $\hat{S}_{\gamma}$. Another example of alias-free sampling is $\left\{t_{n}=\gamma^{-1}\left(\tau_{n}\right)\right\}$, where $\left\{\tau_{n}\right\}$ is Poisson sampling [1].

\section{DISCUSSION}

Image representation by nonuniform sampling schemes is required in a variety of applications in the areas of vision and imaging. In this paper, we have considered a class of images that can be sampled according to a nonuniform sampling scheme, with special emphasis on the case of sampling density that is monotonically decreasing as a function of the radial distance from the center of the area of interest. The class of images under consideration, which have been obtained by a position-varying projection filter, is bandlimited in a compatible Fourier-like domain. Prefiltering in this domain permits decimation of the nonuniform sampling sequence 


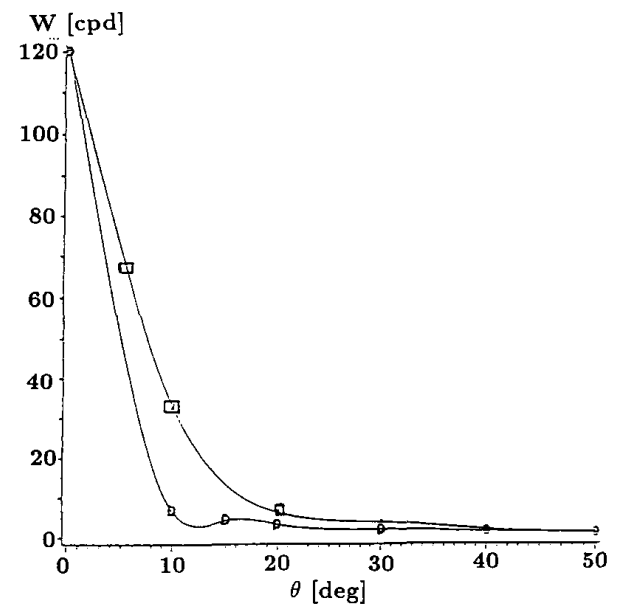

Fig. 7. Position-varying bandwidth as determined by the optical properties of the visual system ( $\square$ ) and by the distribution of cones of the retina (D).

as required, for example, in the process of generating a nonuniform pyramid of image representation.

The notion of alias-free sampling was generalized to apply to a random process with distorted time/position axis, sampled at random points $\left\{t_{n}\right\}$. A sampling sequence is said to be alias-free with respect to the class of random processes under consideration, if there do not exist two processes with different spectral-like densities that yield the same correlation sequence. It was shown that uniformly distributed random jitter over the ideal unwarped sampling grid is alias-free with respect to images that belong to the domain under consideration. This jitter may be caused by misevaluation of the distortion function. Such irregularities in the location of the sampling points are found in the human visual system. This property, which may introduce an error in the reconstructed image, prevents aliasing effects such as the appearance of phantom images and/or jaggedness.

The new approach of the pyramidal representation of the nonuniformly sampled image resembles the properties of the early stages of the visual processing pathway. This approach can be extended through the development of new nonuniform image processing techniques, such as the nonuniform Hough transform for shape analysis with variable distribution of density of features. Development of such nonuniform techniques will in turn facilitate the application of nonuniform systems in machine vision, robotics, or area-of-interest target acquisition systems.

A variety of studies are concerned with understanding how the visual system captures an image. According to the proposed approach, we view the visual system's optics as a position-varying filter that projects the world on the retina. The relation between the optical blur of the visual system [9] and the distribution of cones over the retina [9], [8] are summarized in Fig. 7 in terms of the associated local bandwidth. It turns out that there is a good correspondence in the center and periphery of the visual field. Between 5 and $15^{\circ}$, however, the local bandwidth defined by the optics is much better than the one defined by the cone distribution.

\section{APPENDIX}

Proof of (23): Substituting (22) into (5), we have

$$
\begin{aligned}
\alpha(r, \theta)= & \sum_{n_{1}=-\infty}^{\infty} \sum_{n_{2}=-\infty}^{\infty} \alpha\left(r_{n 1}, \theta_{n 2}\right) \\
& \cdot \operatorname{sinc}\left(\gamma(r)-n_{1}\right) \operatorname{sinc}\left(W \theta-n_{2}\right) .
\end{aligned}
$$

Since $\alpha(r, \theta)=\alpha(r, \theta+2 \pi)$, according to Stark [13], (35) takes the form

$$
\begin{aligned}
\alpha(r, \theta)= & \sum_{n_{1}=-\infty}^{\infty} \sum_{n_{2}=0}^{2 K} \alpha\left(r_{n 1}, \theta_{n_{2}}\right) \operatorname{sinc}\left[\gamma(r)-n_{1}\right] \\
& \cdot \frac{\sin \left[\pi W\left(\theta-\theta_{n_{2}}\right)\right]}{(2 K+1) \sin \left[\left(\theta-\theta_{n_{2}}\right) / 2\right]}
\end{aligned}
$$

as asserted.

Proof of (24): Substituting (22) into (6), the projection operator into $R_{\gamma(r), K}$ is given by

$$
\begin{gathered}
\alpha(r, \theta)=W \int_{-\infty}^{\infty} d \gamma(\rho) \int_{-\infty}^{\infty} d \beta\{\eta(\rho, \beta) \\
\cdot \operatorname{sinc}[\gamma(r)-\gamma(\rho)] \operatorname{sinc}[W(\theta-\beta)]\} .
\end{gathered}
$$

Splitting the integration along the $\beta$ variable into subintervals of $2 \pi$ each, we have

$$
\begin{gathered}
\alpha(r, \theta)=W \int_{-\infty}^{\infty} d \gamma(\rho) \operatorname{sinc}[\gamma(r)-\gamma(\rho)] \\
\sum_{k=-\infty}^{\infty}\left[\int_{2 k \pi}^{2(k+1) \pi} d \beta\{\eta(\rho, \beta) \operatorname{sinc}[W(\theta-\beta)]\}\right]
\end{gathered}
$$

Let $\tilde{\beta}=\beta-2 \pi k$, and then

$$
\begin{gathered}
\int_{2 k \pi}^{2(k+1) \pi} d \beta\{\eta(\rho, \beta) \sin c[W(\theta-\beta)]\}= \\
\int_{0}^{2 \pi} d \tilde{\beta}\{\eta(\rho, \tilde{\beta}+2 \pi k) \operatorname{sinc}[W(\theta-\tilde{\beta}-2 \pi k)]\} .
\end{gathered}
$$

Since $\eta(r, \theta)=\eta(r, \theta+2 \pi k)$ for all $k$, the above integration becomes

$$
\int_{0}^{2 \pi} d \tilde{\beta}\{\eta(\rho, \tilde{\beta}) \operatorname{sinc}[W(\theta-\tilde{\beta}-2 \pi k)]\} .
$$

Substituting (37) into (36) and changing the order of integration and summation, we have

$$
\begin{aligned}
\alpha(r, \theta)= & W \int_{-\infty}^{\infty} \int_{0}^{2 \pi} \eta(\rho, \beta) \operatorname{sinc} \\
& \cdot[\gamma(r)-\gamma(\rho)] g(\theta-\beta) d \beta d \gamma(\rho)
\end{aligned}
$$

where

$$
g(\theta)=\sum_{k} \operatorname{sinc}[W(\theta-2 \pi k)] .
$$

According to Stark [13]

$$
g(\theta)=\frac{\sin [\pi W \theta]}{(2 K+1) \sin (\theta / 2)}
$$

where $W=\frac{2 K+1}{2 \pi}$, as asserted. 


\section{REFERENCES}

[1] F. J. Beutler, "Alias-free randomly timed sampling of stochastic processes," IEEE Trans. Inform. Theory, vol. IT-16, no. 2, Mar. 1970.

[2] P. J. Burt and E. H. Adelson, "The Laplacian pyramid as a compact image code," IEEE Trans. Commun., vol. COM-31, no. 4, pp. 532-540, Apr. 1983.

[3] J. J. Clark, M. R. Palmer, and P. D. Lawrence, "A transformation method for the reconstruction of function from nonuniform spaced samples," IEEE Trans. Acoust., Speech, Signal Processing, vol. ASSP-33, no. 4, pp. 1151-1165, Oct. 1985.

[4] H. D'Angelo, Linear Time-Varying Systems. Boston, MA: Allyn and Bacon, 1970 .

[5] D. E. Dudgeon and R. M. Mersereau, Multidimensional Digital Signal Processing. Englewood Cliffs, NJ: Prentice-Hall, 1984.

[6] J. Hirsch, "Quality of the primate photoreceptor lattice and limits of spatial vision," Vision Res., vol. 24, pp. 347-355, 1984.

[7] H. Horiuchi, "Sampling principle for continuous signals with timevarying bands," Inform. Control. vol. 13, pp. 53-61, July 1968.

[8] R. E. Kronauer and Y. Y. Zeevi, "Reorganization and diversification of signals in vision," IEEE Tran. Syst., Man, Cybern., vol. SMC-15, no. 1, pp. 91-101, 1985.

[9] Y. Le-Grand, Optique Physiologique (Editions de la Revue d'Optique). Paris, 1956, vol. 3

[10] N. Levinson, Gap and Density Theorem. New York: Colloquium Publications, Amer. Math. Soc., 1940, vol. 26.

[11] P. Meer, E. S. Baugher, and A. Rosenfeld, "Frequency domain analysis and synthesis of image pyramid generating kernels," IEEE Trans. Pattern Anal. Machine Intell., vol. PAMI-9, no. 4, July 1987.

[12] M. Porat and Y. Y. Zeevi, "The generalized Gabor scheme of image representation in biological and machine vision," IEEE Trans. Pattern Anal. Machine Intell., vol. 10, no. 4, pp. 452-468, July 1988.

[13] H. Stark, "Sampling theorems in polar coordinates," J. Opt. Soc. Amer., vol. 69, no. 11, pp. 1519-1525, Nov. 1979.

[14] K. Yau and J. B. Thomas, "On some stability and interpolatory properties of nonuniform sampling expansions," IEEE Trans. Circuit Theory, , vol. CT-14, no. 4, pp. 404-408, Dec. 1967.

[15] J. I. Yellott, "Spectral analysis of spatial sampling by photoreceptors: Topological disorder prevents aliasing," Vision Res., vol. 22, pp. 1205-1210, 1982.

[16] _ _ "Spectral consequences of photoreceptor sampling in the rhesus retina," Sci. vol. 221 , pp. 382-385, 1983.

[17] L. A. Zadeh, "A general theory of linear signal transmission systems," J. Franklin Inst., vol. 253, pp. 293-312, 1952.
[18] Y. Y. Zeevi, N. Peterfreund, and E. Shlomot, "Pyramidal image representation in nonuniform systems," in Visual Commun. Image Processing Symp. SPIE, vol. 1001, 1988, pp. 563-571.

[19] Y. Y. Zeevi and E. Shlomot, "Nonuniform sampling and anti-aliasing in image representation," IEEE Trans. Signal Processing, vol. 41, no. 3, pp. 1223-1236, Mar. 1993.

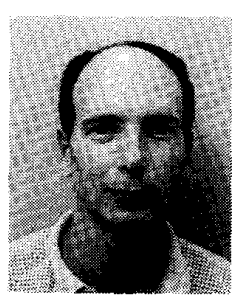

Natan Peterfreund recieved the B.Sc., M.Sc. and D.Sc. degrees in electrical engineering in 1986, 1989, and 1993, respectively, from the Technion-Israel Institute of Technology.

$\mathrm{He}$ is currently a senior research engineer at the Armament Development Authority, Israel. His current research interests are in neural networks, nonlinear systems, and image processing.

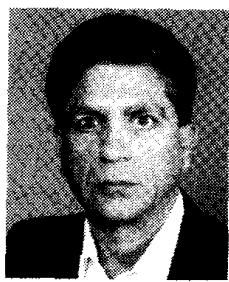

Yehoshua Y. Zeevi received the B.S. degree from the Technion-Israel Institute of Technology, Haifa, Israel, the M.Sc. degree from the University of Rochester, Rochester, NY, and the Ph.D. degree from the University of California, Berkeley.

$\mathrm{He}$ is the Barbara and Norman Seiden Professor of Computer Sciences and the Dean of the Faculty of Electrical Engineering at the Technion. He was a Vinton Hayes Fellow at Harvard University, Cambridge, MA, and has been a regular visitor there. He was also a Visiting Professor at the Massachusetts Institute of Technology, Cambridge, and the CAIP Center of Rutgers University, Piscataway, NJ. His major research is devoted to biological and computer vision, visual communication, and image representation. He is one of the founders of $i$ Sight, Inc, a company devoted to digital video cameras that mimic the visual systems and real-time image processing.

Dr. Zeevi is the Editor-in-Chief of the Journal of Visiual Communication and Image Representation, which is published by Academic Press, and a member of Editorial Boards of several journals. 